\title{
RETICULOCYTOSIS IN THE GUINEA PIG FOLLOWING INJECTIONS OF GASTRIC JUICE AND CONGO RED ${ }^{1}$
}

\author{
By CAMILLE MERMOD \\ (From the Department of Medicine, Stanford University School of Medicine, San Francisco)
}

(Received for publication February 25, 1936)

Various investigators have stated that liver extracts, effective in the treatment of pernicious anemia, cause a rise in the number of reticulocytes in the guinea pig $(1,2,3,4)$. The route of administration (intraperitoneal, subcutaneous, oral) does not affect the type of response nor the height of the reticulocyte rise, but it appears to change slightly the time of the peak of the reticulocytosis. Jacobson (2) defines a positive response as "a rise of the reticulocyte count to at least 2.0 per cent on two successive days, within 6 days after the administration of the substance to be tested." According to his data it falls on the 4th to 7th day following the injection. All the substances tested by him were administered intraperitoneally. Landsberg and Thompson (3) who daily injected commercial liver extract subcutaneously in the abdominal wall obtained a reticulocytosis with peaks between the 5th and 11th days following the beginning of the treatment, most of them falling on the 8th day or later. Miller and Rhoads (4), administering liver extract orally, found the maximum reticulocytosis occurred between the 7 th and 12th days. These data indicate that the antianemic substance, when injected intraperitoneally, produced a reticulocyte peak at an earlier time than when given either subcutaneously in the abdominal wall or orally.

Liver extracts are not the only substances which cause this reticulocytosis in guinea pigs. Miller and Rhoads (4) found that oral administration of extracts of rice polishings and vegex, when incubated with gastric juice, will also give a similar response. The same is also true of gastric juice incubated with beef muscle, with or without pepsin, provided the gastric juice is not heated to $90^{\circ}$ C. before intraperitoneal administration (2).

The following report deals with the reticulocyte response of guinea pigs to the intramuscular administration of gastric juice from patients with

\footnotetext{
1 Supported in part by a grant from the Rockefeller
} Fluid Research Fund. or without pernicious anemia, as well as the response to Congo red and to human gastric juice given intraperitoneally.

\section{METHOD}

Daily reticulocyte counts were made from the blood obtained from an ear vessel of the guinea pig. The blood was received on the corner of a clean number 1 cover slip, and inverted on a slide on which there was an aqueous solution of 1 per cent brilliant cresyl blue in 0.5 per cent sodium citrate and 0.9 per cent sodium chloride. The blood and stain were mixed, the cover slip rimmed with vaseline, and the preparation allowed to stand at least 45 minutes before the reticulocytes were counted. The red cells were examined under oil immersion, at least 1,000 cells being counted in various parts of the cover slip, and the percentage of reticulocytes calculated.

All the material to be tested was introduced by intramuscular injection in a single dose into the thigh of the test animal. The only exceptions to this were the few intraperitoneal injections made of normal gastric juice and of Congo red.

\section{RESULTS}

\section{Control series}

(A) Daily counts were made on 6 non-injected guinea pigs. The average percentage of the reticulocytes varied from 0.85 to 1.62 per cent of the erythrocytes, most of the values falling between 0.9 and 1.2 per cent (Figure 1 and Table I). The greatest change in the reticulocytes was an increase of 90 per cent (limits 0 to 103 per cent) over the lowest value.

$(B)$ Liebig's beef extract was used as a control injection. A solution of the extract was made in distilled water so that $1 \mathrm{cc}$. of the solution represented 0.5 gram of the original material. This amount was injected intramuscularly into 4 guinea pigs. Daily counts for eight consecutive 
TABLE I

Reticulocyte level in uninjected normal guinea pigs on successive days

\begin{tabular}{|c|c|c|c|c|c|c|c|c|c|c|c|c|c|}
\hline \multirow{3}{*}{$\begin{array}{c}\text { Guines } \\
\text { pig } \\
\text { number }\end{array}$} & \multicolumn{13}{|c|}{ Reticulocyte (per cent of R.B.C.) } \\
\hline & \multicolumn{11}{|c|}{ Days } & \multirow{2}{*}{$\begin{array}{c}\text { Day } \\
\text { of } \\
\text { peak }\end{array}$} & \multirow{2}{*}{$\underset{\text { Crease }}{\text { In- }}$} \\
\hline & 1 & 2 & 3 & 4 & 5 & 6 & 7 & 8 & 9 & 10 & 11 & & \\
\hline $\begin{array}{l}\mathbf{5 3 1} \\
\mathbf{5 3 2} \\
533 \\
\mathbf{5 3 4} \\
\mathbf{5 3 7} \\
\mathbf{5 4 0}\end{array}$ & $\begin{array}{l}1.2 \\
0.4 \\
1.4 \\
1.8 \\
1.0 \\
1.6\end{array}$ & $\begin{array}{l}1.0 \\
0.7 \\
2.2 \\
1.5 \\
2.2\end{array}$ & $\begin{array}{l}0.5 \\
0.9 \\
2.0 \\
0.9 \\
1.3 \\
1.2\end{array}$ & $\begin{array}{l}1.9 \\
1.4\end{array}$ & $\begin{array}{l}0.5 \\
1.3 \\
0.8 \\
1.2 \\
1.1\end{array}$ & \begin{tabular}{|l|}
0.6 \\
1.0 \\
2.5 \\
1.8 \\
1.3 \\
1.0
\end{tabular} & $\begin{array}{l}0.4 \\
0.7 \\
2.0 \\
1.5 \\
1.0 \\
1.0\end{array}$ & $\begin{array}{l}0.7 \\
0.4 \\
0.8 \\
0.7 \\
2.1 \\
0.7\end{array}$ & $\begin{array}{l}0.6 \\
0.5 \\
1.7 \\
0.6 \\
1.0 \\
0.8\end{array}$ & $\begin{array}{l}0.8 \\
1.0 \\
1.3 \\
0.5 \\
2.0\end{array}$ & $\begin{array}{l}1.0 \\
0.8 \\
1.5\end{array}$ & $\begin{array}{c}0 \\
4 \\
5 \\
0-5 \\
7 \\
1\end{array}$ & $\begin{array}{r}\text { per cent } \\
0 \\
103 \\
11 \\
0 \\
110 \\
0\end{array}$ \\
\hline Average & 1.23 & 1.52 & 1.13 & 1.62 & .98 & 1.36 & 1.1 & .90 & .85 & 1.12 & 1.1 & 3 & 90 \\
\hline
\end{tabular}

days showed an average reticulocyte range between 0.65 and 1.25 per cent, the latter figure being an increase of 90 per cent over the lower one (Figure 1 and Table II). Of the 2 cases where there was an increase of 500 per cent, all the values, except one, were below 2 per cent and ranged between 0.1 and 1.6 per cent; these values were very similar to those found in uninjected animals. It was concluded that Liebig's beef ex-
TABLE II

Reticulocyte response in guinea pigs following intramuscular injection of Liebig's beef extract

\begin{tabular}{|c|c|c|c|c|c|c|c|c|c|c|c|c|c|c|}
\hline \multirow{3}{*}{$\begin{array}{c}\text { Guines } \\
\text { pig } \\
\text { number }\end{array}$} & \multirow{3}{*}{$\begin{array}{l}\text { Injeo } \\
\text { tion }\end{array}$} & \multicolumn{13}{|c|}{ Reticulocyte (per cent of R.B.C.) } \\
\hline & & \multirow{2}{*}{$\begin{array}{l}\text { Bo- } \\
\text { fore } \\
\text { in- } \\
\text { jec- } \\
\text { tion }\end{array}$} & \multicolumn{10}{|c|}{ Days after injection } & \multirow{2}{*}{$\begin{array}{c}\text { Day } \\
\text { of } \\
\text { peak }\end{array}$} & \multirow{2}{*}{$\underset{\text { crease }}{\text { In- }}$} \\
\hline & & & 1 & 2 & 3 & 4 & 5 & 6 & 7 & 8 & 9 & 10 & & \\
\hline $\begin{array}{l}539 \\
540 \\
541 \\
542\end{array}$ & $\begin{array}{c}\text { grams } \\
0.5 \\
0.5 \\
0.5 \\
0.5\end{array}$ & $\begin{array}{l}0.8 \\
0.1 \\
0.2 \\
2.4\end{array}$ & $\begin{array}{l}0.5 \\
0.3 \\
0.5 \\
1.4\end{array}$ & $\begin{array}{l}0.6 \\
0.6 \\
0.5 \\
1.3\end{array}$ & & $\begin{array}{l}0.5 \\
0.2 \\
1.0 \\
. .9\end{array}$ & $\begin{array}{l}1.0 \\
0.5 \\
1.6 \\
1.9\end{array}$ & $\begin{array}{l}1.5 \\
0.2 \\
0.7 \\
1.6\end{array}$ & & $\begin{array}{l}0.9 \\
0.7 \\
1.3 \\
1.1\end{array}$ & & $\begin{array}{l}0.4 \\
1.3 \\
2.2 \\
0.7\end{array}$ & $\begin{array}{r}6 \\
10 \\
10\end{array}$ & $\begin{array}{c}\text { per cent } \\
130 \\
550 \\
528\end{array}$ \\
\hline \multicolumn{2}{|c|}{ Average } & .87 & .67 & .75 & & .65 & 1.25 & 1.0 & & 1.0 & & 1.15 & 5 & 43 \\
\hline
\end{tabular}

tract contains no substance reticulocytogenic for the guinea pig. This agrees perfectly with clinical experience, since beef extract has not been shown to possess any curative potency in pernicious anemia.

The average degree of rise of the reticulocytes following the injection of beef extract was practically the same as that found in normal uninjected guinea pigs. From these values it was decided

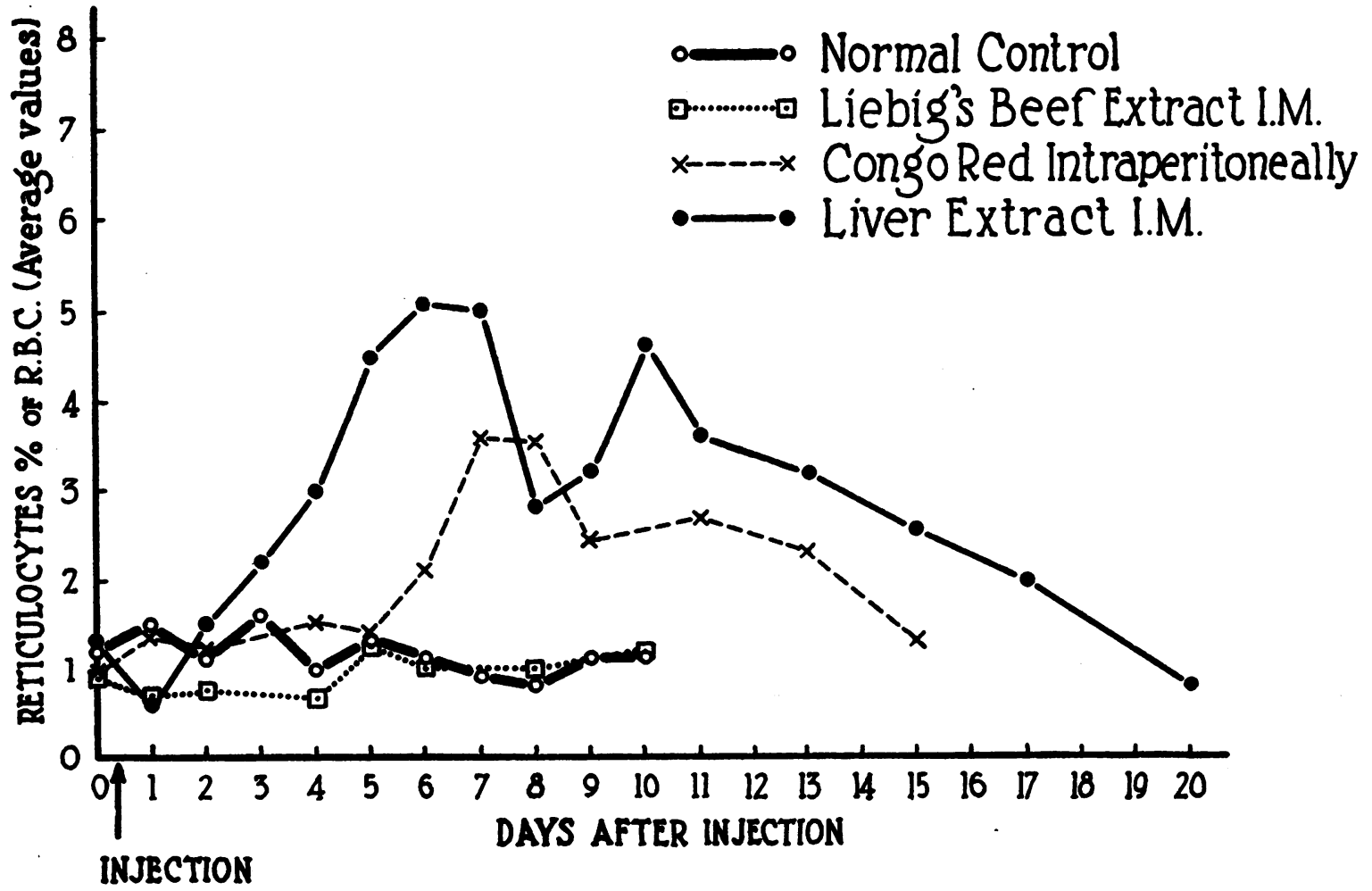

Fig. 1. Reticulocyte Level in Uninjected Guinea Pigs and Response to Injections of Beef Extract, Congo Red, Liver Extract 
that in order to be called reticulocytogenic for the guinea pig a substance must produce within eight days following the first injection an increase of the reticulocytes greater than 100 per cent of the control value, and with an absolute reticulocyte value greater than 2 per cent of the erythrocytes. Since the reaction did not begin before the 2 nd day in any instance, the average of the first two counts (day of injection and first day following) was taken as the control reticulocyte value. The graphs are based on the average values obtained from a number of animals undergoing the same treatment.

\section{Liver extract}

One-tenth cubic centimeter of commercial antipernicious anemia liver extract (equivalent to 3.3 grams of whole liver) was injected intramuscularly into the guinea pigs. About 85 per cent of the animals gave a positive response (Table III). The rise of the reticulocytes began on the second day following the injection and reached its peak

TABLE III

Reticulocyte response in guinea pigs following intramuscular injection of antipernicious anemia liver extract

\begin{tabular}{|c|c|c|c|c|c|c|c|c|c|c|c|c|c|c|c|}
\hline \multirow{2}{*}{$\begin{array}{l}\text { Guinea pig } \\
\text { number }\end{array}$} & \multirow{2}{*}{$\begin{array}{c}\text { Before } \\
\text { injec- } \\
\text { tion }\end{array}$} & \multicolumn{12}{|c|}{ Days after injection } & \multirow{2}{*}{$\begin{array}{c}\text { Day } \\
\text { of } \\
\text { peak }\end{array}$} & \multirow{2}{*}{$\begin{array}{c}\text { In- } \\
\text { crease }\end{array}$} \\
\hline & & 1 & 2 & 3 & 4 & 5 & 6 & 7 & 8 & 9 & 10 & 11 & 12 & & \\
\hline & & & & & & & & & & & & & & & per cent \\
\hline
\end{tabular}

Injection of 1 cc. Lilly L.E. (3.3 grams liver)

\begin{tabular}{|c|c|c|c|c|c|c|c|c|c|c|c|c|c|c|c|}
\hline $\begin{array}{l}\mathbf{5 3 1} \\
\mathbf{5 3 2} \\
\mathbf{5 3 3} \\
\mathbf{5 3 6} \\
\mathbf{5 4 1} \\
\mathbf{5 4 2} \\
\mathbf{5 4 4} \\
\mathbf{5 4 9} \\
\mathbf{5 5 0} \\
\mathbf{5 5 1} \\
\mathbf{5 5 2} \\
\mathbf{5 5 3} \\
\mathbf{5 5 4} \\
\mathbf{5 5 5} \\
\mathbf{5 5 7} \\
\mathbf{5 5 8} \\
\mathbf{5 5 9} \\
\mathbf{5 6 0} \\
\mathbf{5 6 1} \\
\mathbf{5 6 4} \\
\mathbf{5 7 2} \\
\mathbf{5 7 3} \\
\mathbf{5 7 5}\end{array}$ & $\begin{array}{l}0.9 \\
1.8 \\
1.6 \\
0.9 \\
2.3 \\
0.8 \\
1.1 \\
1.6 \\
1.3 \\
2.4 \\
1.7 \\
1.0 \\
2.3 \\
1.7 \\
2.5 \\
0.5 \\
0.5 \\
0.9 \\
0.6 \\
1.5 \\
1.1 \\
2.1 \\
3.4\end{array}$ & 1.0 & $\begin{array}{l}1.4 \\
2.2 \\
1.0 \\
2.1 \\
1.8 \\
1.5 \\
1.7 \\
2.7 \\
0.7 \\
2.1 \\
0.9 \\
2.5 \\
3.2 \\
1.3 \\
0.2 \\
0.7 \\
1.0 \\
1.0 \\
1.3 \\
1.4 \\
1.8 \\
2.7\end{array}$ & $\begin{array}{l}1.7 \\
4.0 \\
0.9 \\
2.3 \\
\\
2.2 \\
1.6 \\
8.1 \\
1.6 \\
1.9 \\
4.5 \\
1.0 \\
3.6 \\
1.7 \\
1.0 \\
1.2 \\
1.6 \\
0.8 \\
2.0 \\
3.0 \\
1.9 \\
2.1\end{array}$ & $\begin{array}{l}1.4 \\
3.2 \\
1.0 \\
2.3 \\
4.0 \\
2.8 \\
2.2 \\
8.5 \\
2.0 \\
5.2 \\
4.2 \\
0.8 \\
4.6 \\
6.0 \\
1.7 \\
3.2 \\
4.2 \\
2.2 \\
1.0 \\
2.3 \\
2.6 \\
3.9 \\
2.5\end{array}$ & $\begin{array}{r}3.0 \\
5.8 \\
5.4 \\
4.0 \\
7.2 \\
4.0 \\
3.6 \\
5.5 \\
5.0 \\
10.5 \\
5.9 \\
1.8 \\
3.6 \\
4.9 \\
1.4 \\
7.2 \\
1.4 \\
1.8 \\
1.6 \\
3.4 \\
7.0 \\
3.8 \\
1.8\end{array}$ & $\begin{array}{l}3.8 \\
2.8 \\
2.6 \\
3.0 \\
5.0 \\
4.8 \\
3.0 \\
9.8 \\
8.4 \\
3.8 \\
3.4 \\
3.4 \\
4.8 \\
3.6 \\
5.6 \\
7.0 \\
1.8 \\
2.6 \\
2.4 \\
5.6 \\
7.8 \\
6.6 \\
9.8\end{array}$ & $\begin{array}{r}4.2 \\
2.8 \\
2.6 \\
0.8 \\
2.6 \\
1.0 \\
3.0 \\
5.2 \\
7.4 \\
6.2 \\
5.0 \\
1.6 \\
3.6 \\
1.2 \\
4.8 \\
2.4 \\
8.8 \\
3.2 \\
3.6 \\
4.0 \\
5.0 \\
6.4 \\
13.0\end{array}$ & $\begin{array}{l}2.0 \\
4.0 \\
2.4 \\
2.6 \\
5.4 \\
2.8 \\
2.4 \\
1.2 \\
2.8 \\
0.2 \\
2.6 \\
0.2 \\
1.2 \\
0.6 \\
1.0 \\
1.2 \\
1.2 \\
1.0 \\
2.0 \\
2.0 \\
3.2 \\
3.0\end{array}$ & 2.2 & $\begin{array}{l}1.0 \\
1.8 \\
0.8 \\
1.6 \\
1.2 \\
0.4 \\
1.2\end{array}$ & $\begin{array}{l}3.2 \\
1.4 \\
1.4 \\
2.8 \\
\\
0.8 \\
3.0 \\
2.1\end{array}$ & & $\begin{array}{l}7 \\
5 \\
5 \\
5 \\
5 \\
6 \\
5 \\
6 \\
6 \\
5 \\
5 \\
6 \\
6 \\
4 \\
7 \\
5 \\
7 \\
7 \\
7 \\
6 \\
6 \\
6 \\
7\end{array}$ & $\begin{array}{r}366 \\
222 \\
235 \\
345 \\
213 \\
500 \\
225 \\
510 \\
550 \\
340 \\
250 \\
240 \\
109 \\
250 \\
125 \\
1340 \\
1660 \\
235 \\
500 \\
275 \\
610 \\
214 \\
280\end{array}$ \\
\hline \multicolumn{16}{|c|}{ Injection of 0.1 cc. Lilly L.E. (3.3 grams liver) } \\
\hline $\begin{array}{l}\text { OBA } \\
\text { OBB }\end{array}$ & $\begin{array}{l}0.6 \\
1.1\end{array}$ & 0.9 & 0.2 & 0.4 & $\begin{array}{l}2.0 \\
1.5\end{array}$ & $\begin{array}{l}3.2 \\
8.6\end{array}$ & $\begin{array}{l}7.2 \\
7.0\end{array}$ & $\begin{array}{r}10.7 \\
4.4\end{array}$ & 5.0 & 1.6 & & 1.2 & & $\begin{array}{l}7 \\
5\end{array}$ & $\begin{array}{r}1680 \\
540\end{array}$ \\
\hline \multicolumn{16}{|c|}{ Injection of $0.1 \mathrm{cc}$. Lederle (3.3 grams liver) } \\
\hline $\begin{array}{l}1 \\
3 \\
4\end{array}$ & $\begin{array}{l}0.1 \\
0.1 \\
0.1\end{array}$ & $\begin{array}{l}0.5 \\
0.1 \\
0.2\end{array}$ & $\begin{array}{l}0.9 \\
0.3 \\
0.3\end{array}$ & $\begin{array}{l}2.7 \\
3.0 \\
0.5\end{array}$ & $\begin{array}{l}2.8 \\
3.2 \\
2.0\end{array}$ & 3.2 & $\begin{array}{l}3.2 \\
2.9\end{array}$ & $\begin{array}{r}6.3 \\
10.0 \\
2.5\end{array}$ & $\begin{array}{l}9.4 \\
3.6 \\
6.0\end{array}$ & $\begin{array}{l}2.5 \\
7.4 \\
3.0\end{array}$ & $\begin{array}{l}3.7 \\
5.1 \\
4.1\end{array}$ & $\begin{array}{l}5.0 \\
6.1 \\
3.5\end{array}$ & $\begin{array}{l}1.7 \\
4.3\end{array}$ & $\begin{array}{l}8 \\
6 \\
8\end{array}$ & $\begin{array}{l}3030 \\
9900 \\
3900\end{array}$ \\
\hline Average & 1.3 & 0.58 & 1.52 & 2.22 & 2.97 & 4.45 & 5.06 & 4.49 & 2.8 & 3.3 & 1.64 & 2.62 & 3.0 & 6 & 440 \\
\hline
\end{tabular}


on the 6th day (Individual limits of the peak 4th to 8th day). The average percentage rise was 440 per cent, ranging from 109 to 3030 per cent (Figure 1).

The ability of guinea pigs to respond to intramuscular injections of liver extract was used as a means of dividing them into two groups : reacting animals (those showing a reticulocytosis after the treatment) and non-reacting ones (those showing no reticulocytosis after treatment). Only reacting animals were used in subsequent experiments. Values obtained with 28 animals are found in Figure 1 and Table III.

When a second injection was made on the 12 th to 18th day following the first one, no second reticulocytosis was obtained. This absence of a second reaction showed that a maximal effect had been obtained with the first injection, and was also used as a criterion of the activity of the materials tested.

\section{Congo red}

A 1.5 per cent solution of Congo red (Coleman and Bell), in 6 per cent glucose, was injected intraperitoneally into 6 guinea pigs. The total
TABLE IV

Reticulocyte response in guinea pigs following intraperitoneal injection of 1.5 per cent Congo red solution

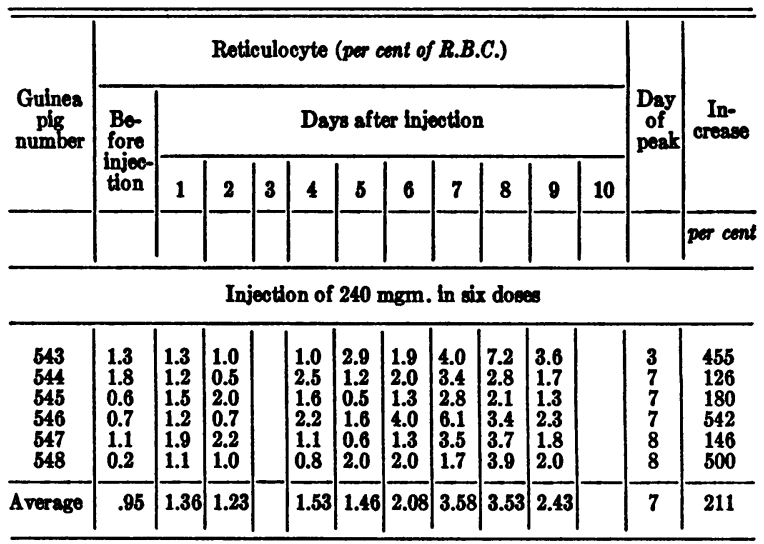

quantity injected into each animal (240 mgm.) was divided into 6 doses. No toxic manifestations followed the administration of the dye. The peak of the reticulocytosis occurred on the 6 th to 7 th day, with a rise of 260 per cent over the initial value (Figure 1 and Table IV). There was no second reticulocytosis following an injection of 0.3 cc. of liver extract intramuscularly on the 15 th day.

TABLE V

Reticulocyte response in guinea pigs following intramuscular injection of normal gastric juice

\begin{tabular}{|c|c|c|c|c|c|c|c|c|c|c|c|c|c|c|c|c|}
\hline \multirow{3}{*}{$\begin{array}{c}\text { Guinea } \\
\text { pig } \\
\text { number }\end{array}$} & \multirow{3}{*}{ Injection } & \multicolumn{13}{|c|}{ Reticulocyte (per cent of R.B.C.) } & \multirow{3}{*}{$\begin{array}{c}\text { Day } \\
\text { of } \\
\text { peak }\end{array}$} & \multirow{3}{*}{ Increase } \\
\hline & & \multirow{2}{*}{$\begin{array}{c}\text { Before } \\
\text { injection }\end{array}$} & \multicolumn{12}{|c|}{ Days after injection } & & \\
\hline & & & 1 & 2 & 3 & 4 & 5 & 6 & 7 & 8 & 9 & 10 & 11 & 12 & & \\
\hline $\begin{array}{r}1 \\
2 \\
3 \\
3 \\
\mathbf{4} \\
\mathbf{5} \\
\mathbf{5 3 1} \\
\mathbf{5 3 3} \\
\mathbf{5 3 7} \\
\mathbf{5 4 0} \\
\mathbf{5 4 2} \\
\mathbf{5 5 0} \\
\mathbf{5 5 0} \\
\mathbf{5 5 1} \\
\mathbf{5 5 2} \\
\mathbf{5 5 3} \\
\mathbf{5 5 4} \\
\mathbf{5 5 7} \\
\mathbf{5 7 3} \\
\mathbf{5 7 4}\end{array}$ & $\begin{array}{l}4 \text { cc. S. } \\
4 \text { cc. S. } \\
4 \text { cc. S. } \\
0.2 \text { cc. B. } \\
1 \text { cc. S. } \\
1 \text { cc. S. } \\
0.05 \text { cc. Mo. } \\
0.01 \text { cc. Mo. } \\
0.025 \text { cc. Mo. } \\
0.1 \text { cc. no. } 2 \\
0.5 \text { cc. no. } 2 \\
0.05 \text { cc. Mo. } \\
0.3 \text { cc. Ze. } \\
0.1 \text { cc. Mo. } \\
0.025 \text { cc. Mo. } \\
0.05 \text { cc. Mo. } \\
0.5 \text { cc. Sb. } \\
0.02 \text { cc. Sm. } \\
0.25 \text { cc. O. }\end{array}$ & $\begin{array}{l}2.1 \\
2.5 \\
2.1 \\
1.6 \\
1.5 \\
3.4 \\
1.7 \\
0.9 \\
1.8 \\
1.0 \\
1.2 \\
0.4 \\
0.9 \\
0.6 \\
0.7 \\
2.4 \\
1.4 \\
2.0 \\
1.2 \\
1.6\end{array}$ & 2.5 & $\begin{array}{l}1.7 \\
2.5 \\
2.4 \\
3.4 \\
1.8 \\
3.6\end{array}$ & $\begin{array}{l}1.7 \\
2.9 \\
3.9 \\
1.2 \\
3.3 \\
3.4 \\
3.1 \\
2.5 \\
\\
1.9 \\
1.3 \\
1.8 \\
4.2 \\
2.0 \\
0.4 \\
1.5\end{array}$ & $\begin{array}{l}1.6 \\
\\
3.0 \\
2.8 \\
3.1 \\
\\
3.6 \\
1.8 \\
1.2 \\
1.7 \\
3.7 \\
2.4 \\
0.8 \\
0.6 \\
1.7\end{array}$ & \begin{tabular}{|l|}
1.8 \\
3.6 \\
2.6 \\
5.1 \\
1.1 \\
5.8 \\
2.3 \\
4.6 \\
2.0 \\
1.8 \\
1.6 \\
8.0 \\
3.2 \\
1.6 \\
3.5 \\
2.3 \\
3.6 \\
2.1 \\
1.1 \\
2.9
\end{tabular} & $\begin{array}{l}4.3 \\
4.7 \\
6.2 \\
3.1 \\
4.9 \\
8.4 \\
1.8 \\
4.0 \\
4.2 \\
2.9 \\
3.1 \\
3.8 \\
7.1 \\
3.3 \\
2.0 \\
7.9 \\
4.8 \\
6.4 \\
3.8 \\
4.1\end{array}$ & $\begin{array}{l}4.0 \\
9.8 \\
7.4 \\
1.0 \\
6.0 \\
8.6 \\
4.0 \\
6.0 \\
5.1 \\
5.2 \\
4.2 \\
1.7 \\
4.2 \\
1.2 \\
2.1 \\
3.2 \\
1.0 \\
3.2 \\
2.6 \\
4.0\end{array}$ & $\begin{array}{r}6.4 \\
4.6 \\
4.0 \\
2.8 \\
2.3 \\
12.4 \\
5.4 \\
8.0 \\
4.6 \\
4.6 \\
7.2 \\
2.1 \\
1.3 \\
2.5 \\
2.4 \\
1.5\end{array}$ & $\begin{array}{r}7.4 \\
3.6 \\
3.8 \\
4.8 \\
3.8 \\
10.8 \\
1.2 \\
5.2 \\
4.4 \\
1.4 \\
2.7 \\
2.3 \\
1.0 \\
\\
1.0\end{array}$ & & 0.8 & $\begin{array}{l}3.4 \\
2.4 \\
1.4 \\
1.4\end{array}$ & $\begin{array}{l}9 \\
7 \\
7 \\
5 \\
7 \\
8 \\
8 \\
8 \\
7 \\
7 \\
8 \\
5 \\
6 \\
6 \\
5 \\
6 \\
6 \\
6 \\
6 \\
6\end{array}$ & $\begin{array}{c}\text { per cent } \\
252 \\
292 \\
252 \\
\\
300 \\
265 \\
218 \\
230 \\
183 \\
420 \\
500 \\
1900 \\
688 \\
450 \\
400 \\
230 \\
240 \\
220 \\
216 \\
156\end{array}$ \\
\hline \multicolumn{2}{|r|}{ Average } & 1.65 & & 2.02 & 2.34 & 2.15 & 3.03 & 4.54 & 4.22 & 4.50 & 3.81 & & & 1.98 & & 174 \\
\hline
\end{tabular}


TABLE VI

Reticulocyte response in guinea pigs following intraperitoneal injection of normal gastric juice

\begin{tabular}{|c|c|c|c|c|c|c|c|c|c|c|c|c|c|c|c|}
\hline \multirow{3}{*}{$\begin{array}{c}\text { Guinea } \\
\text { pig } \\
\text { num- } \\
\text { ber }\end{array}$} & \multirow{3}{*}{ Injection } & \multicolumn{12}{|c|}{ Reticulocyte (per cent of R.B.C.) } & \multirow{3}{*}{$\begin{array}{c}\text { Day } \\
\text { of } \\
\text { peak }\end{array}$} & \multirow{3}{*}{$\begin{array}{c}\text { In- } \\
\text { crease }\end{array}$} \\
\hline & & \multirow{2}{*}{$\begin{array}{c}\text { Before } \\
\text { injec- } \\
\text { tion }\end{array}$} & \multicolumn{11}{|c|}{ Days after injection } & & \\
\hline & & & 1 & 2 & 3 & 4 & 5 & 6 & 7 & 8 & 9 & 10 & 11 & & \\
\hline $\begin{array}{l}\mathbf{5 4 9} \\
\mathbf{5 5 0} \\
\mathbf{5 5 4} \\
\mathbf{5 5 7} \\
\mathbf{5 6 4} \\
\mathbf{5 6 5} \\
\mathbf{5 6 6} \\
\mathbf{5 7 4}\end{array}$ & $\begin{array}{l}0.5 \text { cc. Bo. } \\
0.5 \text { cc. Bo. } \\
0.5 \text { cc. no. } 2 \\
0.5 \text { cc. no. } 2 . \\
0.1 \text { cc. } \text { Mo. } \\
0.05 \text { cc. } \text { Mo. } \\
0.025 \text { cc. } \text { Mo. } \\
0.5 \text { cc. } \text { Sm. }\end{array}$ & $\begin{array}{l}1.2 \\
2.2 \\
0.5 \\
0.4 \\
1.5 \\
1.1 \\
1.5 \\
1.6\end{array}$ & $\begin{array}{l}1.1 \\
1.4 \\
1.1 \\
1.0\end{array}$ & $\begin{array}{l}1.7 \\
2.1 \\
1.1\end{array}$ & $\begin{array}{l}0.6 \\
1.6 \\
0.5 \\
1.0 \\
0.9 \\
1.3 \\
2.8 \\
1.2\end{array}$ & $\begin{array}{l}1.3 \\
2.0 \\
1.3 \\
1.2 \\
1.6 \\
0.7 \\
1.9 \\
0.6\end{array}$ & $\begin{array}{l}0.5 \\
1.4 \\
1.0 \\
2.4 \\
1.2 \\
2.0 \\
1.8 \\
1.2\end{array}$ & $\begin{array}{l}2.2 \\
1.0 \\
1.2 \\
1.7\end{array}$ & $\begin{array}{l}1.3 \\
1.0 \\
1.7 \\
2.2 \\
3.6 \\
0.6 \\
1.0 \\
1.2\end{array}$ & $\begin{array}{l}1.8 \\
0.6 \\
3.4 \\
1.9 \\
2.6 \\
2.4 \\
1.2\end{array}$ & $\begin{array}{l}2.7 \\
0.9 \\
3.8 \\
2.0 \\
2.4 \\
1.8 \\
1.6\end{array}$ & 0.4 & $\begin{array}{l}3.9 \\
1.1 \\
2.9 \\
2.5 \\
2.2 \\
1.4 \\
0.2\end{array}$ & $\begin{array}{r}11 \\
9 \\
11 \\
7 \\
8 \\
3 \\
6\end{array}$ & $\begin{array}{c}\text { per cent } \\
225 \\
263 \\
256 \\
140 \\
120 \\
87 \\
8\end{array}$ \\
\hline & Average & 1.25 & 1.15 & 1.63 & 1.23 & 1.32 & 1.43 & 1.52 & 1.57 & 1.64 & 2.17 & & 2.02 & 9 & 73 \\
\hline
\end{tabular}

\section{Gastric juice}

Filtered, unneutralized gastric juice from normal individuals was injected intramuscularly and intraperitoneally into reacting animals, 2 months or more after a liver injection. Only one injection was given, varying in amount from $4 \mathrm{cc}$. to $0.01 \mathrm{cc}$. The larger doses of gastric juice were injected undiluted, but a dilution of $1: 10$ was made in the case of the smaller amounts in order to be able to measure the quantities more accurately.

(a) Intramuscular injections: Following the intramuscular injection the reticulocytes of the guinea pigs remained at the preinjection level until about the third or fourth day, after which they began to rise, reaching their peak from the 5th to the 8th day following the injection (Figure 2 and Table V). The average increase

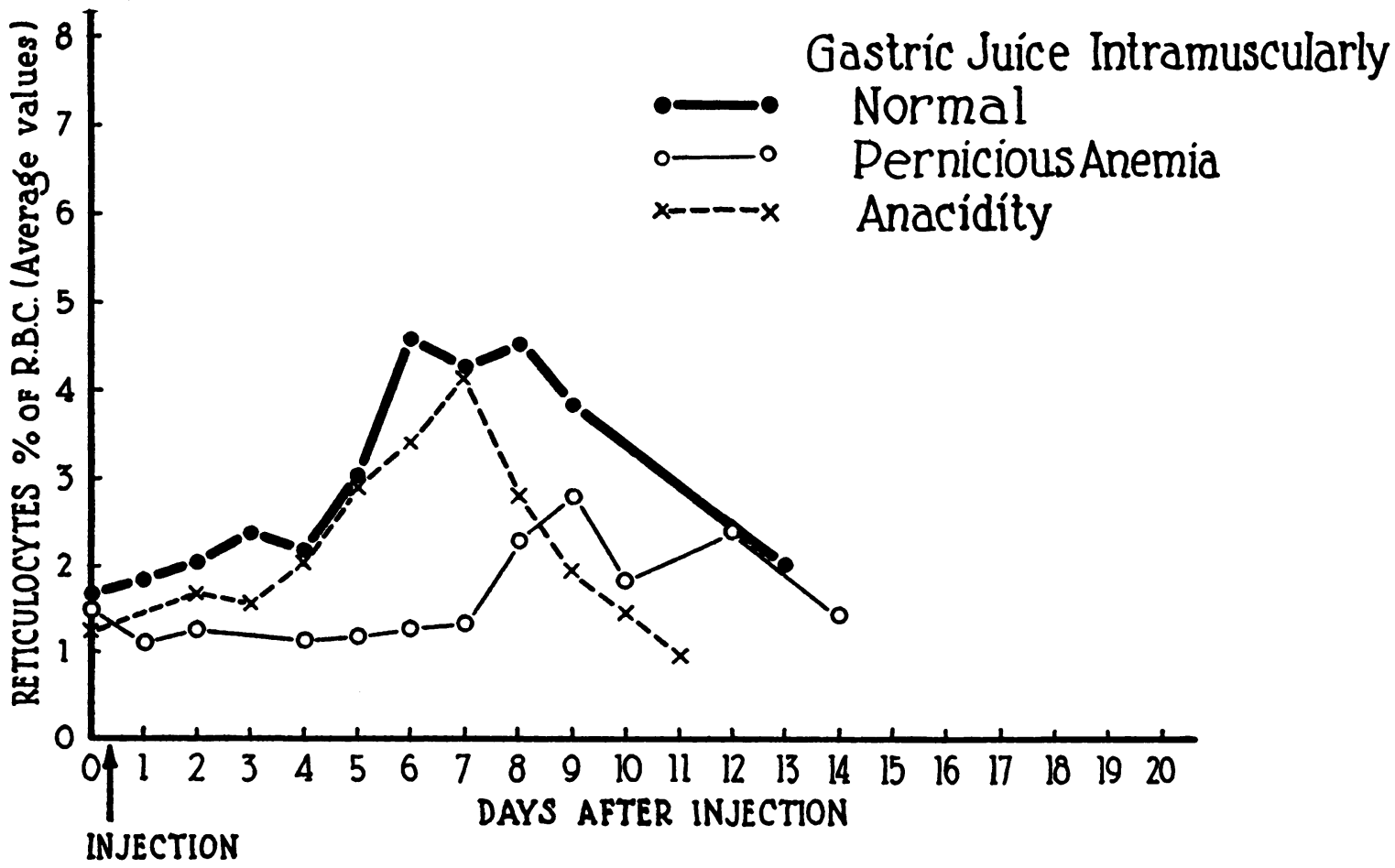

Fig. 2. Reticulocyte Response of Guinea Pigs to Intramuscular Injections of Gastric Tutce 
TABLE VII

Reticulocyte response in guinea pigs following intramuscular injection of anacidity gastric juice

\begin{tabular}{|c|c|c|c|c|c|c|c|c|c|c|c|c|c|c|c|}
\hline \multirow{3}{*}{$\begin{array}{c}\text { Guinea } \\
\text { pig } \\
\text { num- } \\
\text { ber }\end{array}$} & \multirow{3}{*}{ Injection } & \multicolumn{12}{|c|}{ Reticulocyte (per cent of R.B.C.) } & \multirow{3}{*}{$\begin{array}{c}\text { Day } \\
\text { of } \\
\text { peak }\end{array}$} & \multirow{3}{*}{$\begin{array}{c}\text { In- } \\
\text { crease }\end{array}$} \\
\hline & & \multirow{2}{*}{$\begin{array}{c}\text { Before } \\
\text { injec- } \\
\text { tion }\end{array}$} & \multicolumn{11}{|c|}{ Days after injection } & & \\
\hline & & & 1 & 2 & 3 & 4 & 5 & 6 & 7 & 8 & 9 & 10 & 11 & & \\
\hline $\begin{array}{l}\mathbf{5 3 8} \\
\mathbf{5 3 6} \\
\mathbf{5 4 1} \\
\mathbf{5 4 4} \\
\mathbf{5 4 8} \\
\mathbf{5 5 5} \\
\mathbf{5 5 6} \\
\mathbf{5 5 7} \\
\mathbf{5 5 8} \\
\mathbf{5 6 0} \\
\mathbf{5 6 1} \\
\mathbf{5 6 2} \\
\mathbf{5 6 3}\end{array}$ & $\begin{array}{l}0.02 \text { cc. } \mathrm{E} . \\
0.5 \text { cc. } \mathrm{Me} . \\
0.02 \text { cc. } \mathrm{E} . \\
0.1 \text { cc. } \mathrm{Ho} . \\
0.01 \text { cc. } \mathrm{E} . \\
1 \mathrm{cc} . \mathrm{Hu} . \\
0.5 \mathrm{cc} . \mathrm{Hu} \text {. } \\
0.1 \text { cc. Hu. } \\
1 \text { cc. } \mathrm{L} . \\
0.1 \text { cc. } \mathrm{L} . \\
0.5 \text { cc. } \mathrm{E} . \\
0.1 \text { cc. } \mathrm{E} . \\
0.02 \text { cc. } \mathrm{E} .\end{array}$ & $\begin{array}{l}1.2 \\
0.2 \\
1.9 \\
1.0 \\
1.5 \\
0.9 \\
1.0 \\
1.1 \\
1.2 \\
2.4 \\
0.5 \\
1.1 \\
1.8\end{array}$ & & $\begin{array}{l}3.5 \\
2.0 \\
1.8 \\
0.6 \\
2.1 \\
0.6 \\
0.5 \\
2.2 \\
1.3\end{array}$ & $\begin{array}{l}0.9 \\
0.6 \\
\\
0.9 \\
2.8 \\
0.5 \\
1.4 \\
3.2 \\
1.0 \\
1.8 \\
2.2\end{array}$ & $\begin{array}{l}4.0 \\
3.4 \\
1.2 \\
\\
1.9 \\
0.3 \\
2.8 \\
1.0 \\
\\
\\
\\
1.8 \\
1.7 \\
2.4\end{array}$ & $\begin{array}{l}3.0 \\
6.3 \\
3.7 \\
2.7 \\
3.3 \\
0.5 \\
6.0 \\
1.2 \\
3.0 \\
1.8 \\
1.2 \\
2.5 \\
3.1\end{array}$ & $\begin{array}{l}3.7 \\
3.7 \\
4.3 \\
2.2 \\
3.6 \\
1.8 \\
5.0 \\
2.7 \\
4.0 \\
4.9 \\
1.8 \\
2.8 \\
3.8\end{array}$ & $\begin{array}{l}2.7 \\
9.4 \\
4.3 \\
2.7 \\
2.2 \\
3.2 \\
6.7 \\
5.0 \\
3.7 \\
6.0 \\
2.0 \\
3.2 \\
2.6\end{array}$ & $\begin{array}{l}2.0 \\
1.6 \\
0.8 \\
4.6 \\
3.2\end{array}$ & $\begin{array}{l}1.2 \\
5.0 \\
0.6 \\
1.5 \\
\\
0.3 \\
5.4 \\
2.4 \\
\\
\\
0.4 \\
1.4 \\
1.0\end{array}$ & $\begin{array}{l}1.0 \\
2.2 \\
1.0 \\
2.6 \\
0.3\end{array}$ & $\begin{array}{l}1.2 \\
0.8 \\
\\
0.4 \\
0.7 \\
0.9 \\
1.1\end{array}$ & $\begin{array}{c}4 \\
7 \text { to } 8 \\
6 \text { to } 7 \\
7 \\
6 \\
7 \\
7 \\
7 \\
6 \\
7 \\
7 \\
8 \\
6\end{array}$ & $\begin{array}{c}\text { per cent } \\
235 \\
3700 \\
125 \\
170 \\
140 \\
255 \\
570 \\
355 \\
230 \\
150 \\
300 \\
320 \\
115\end{array}$ \\
\hline & rage & 1.21 & & 1.62 & 1.53 & 2.05 & 2.94 & 3.40 & 4.13 & 2.78 & 1.92 & 1.42 & .96 & 7 & 240 \\
\hline
\end{tabular}

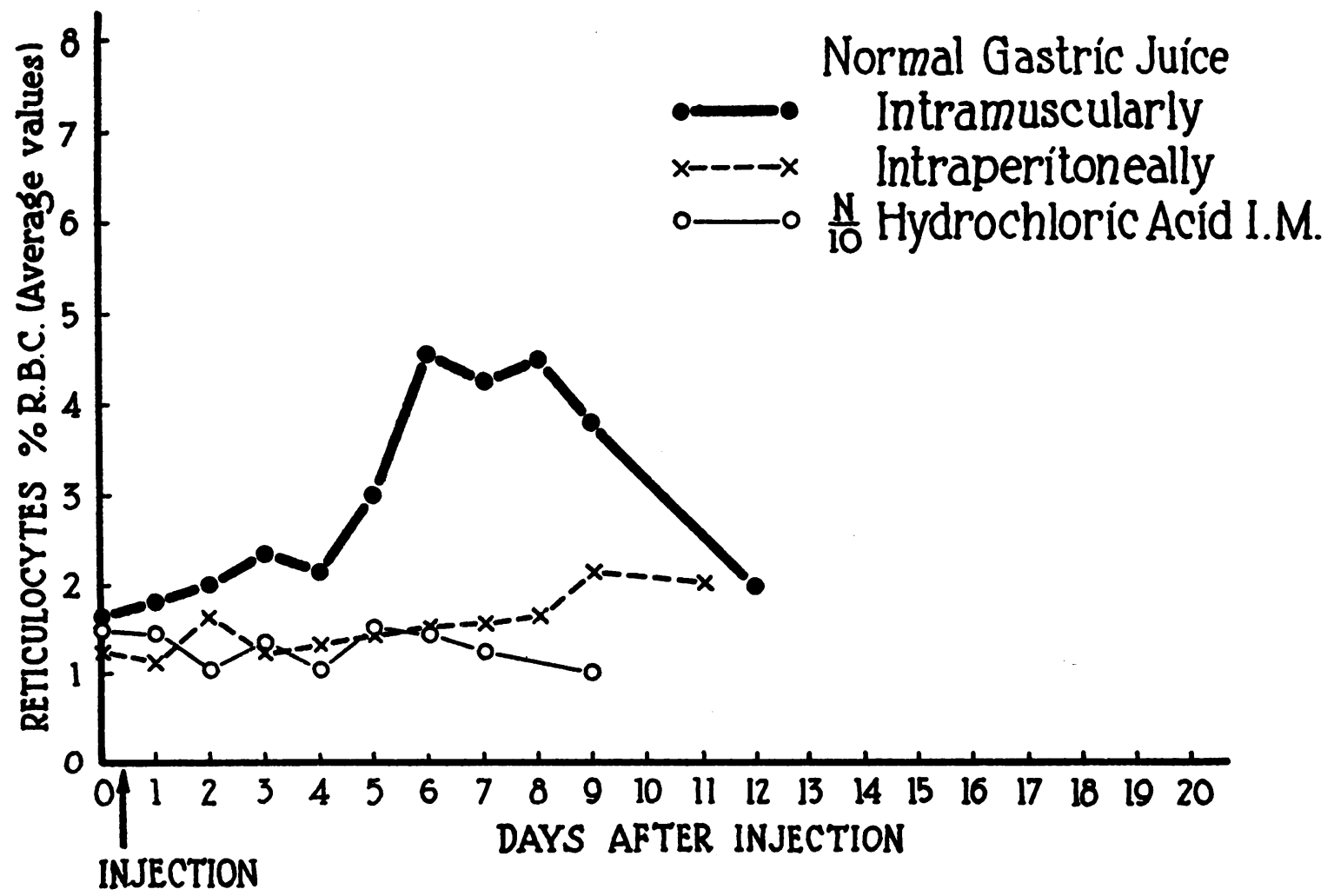

Fig. 3. Reticulocyte Response of Guinea Pigs to Intramuscular and Intraperitoneal Injections of Gastric Juice; and Intramuscular Injection of Hydrochloric Acid 
RETICULOCYTOSIS IN GUINEA PIGS

TABLE VIII

Reticulocyte response in guinea pigs following intramuscular injection of gastric juice from pernicious anemia patients

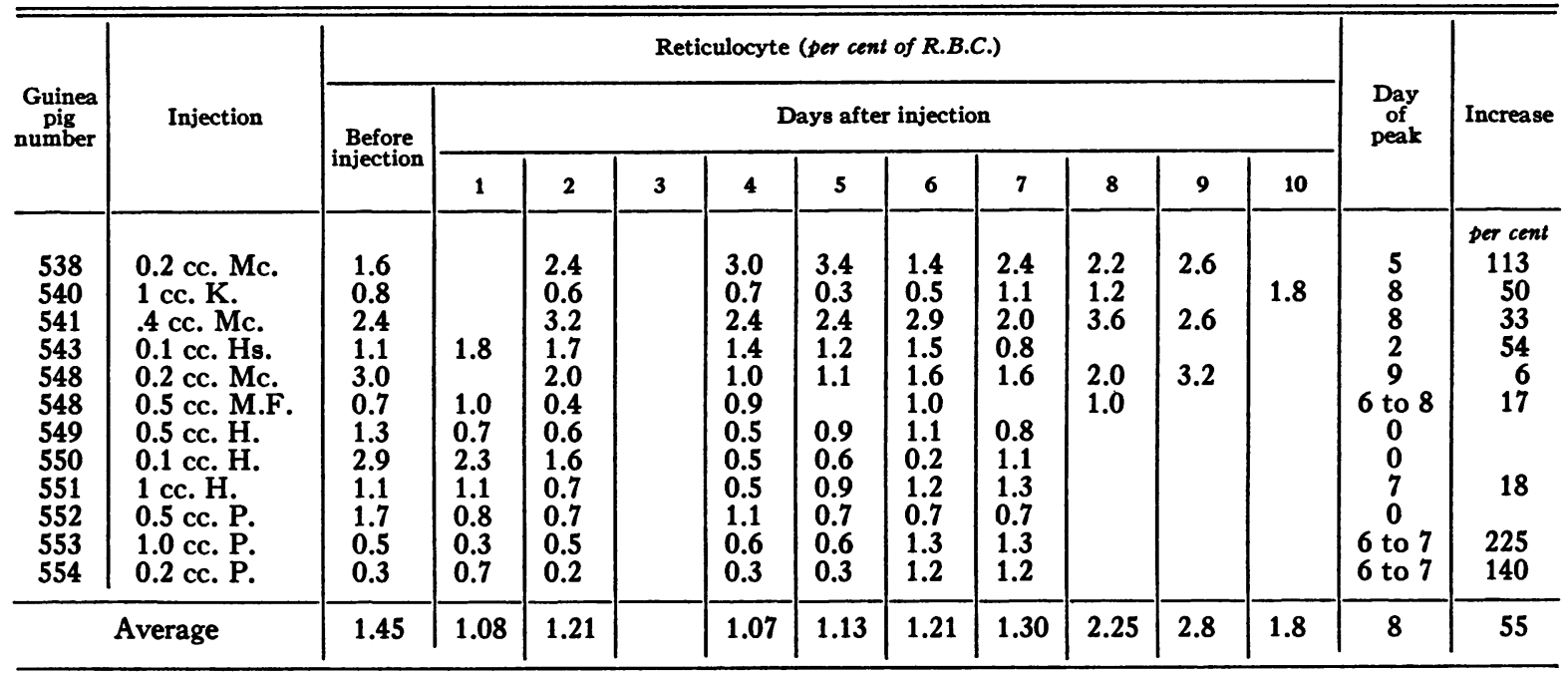

was 175 per cent. The per cent of rise of reticulocytes did not increase with the larger doses, and was approximately the same whether $4 \mathrm{cc}$. or $0.02 \mathrm{cc}$. of gastric juice were injected. The curve resembled closely that obtained following the intramuscular injection of liver extract. The injection of liver extract intramuscularly did not cause any further increase of reticulocytes.

(b) Intraperitoneal injections. Normal gastric juice, giving good responses when injected intramuscularly in doses as low as 0.02 cc., caused only slight and rather late (7th to 11 th day) rise in reticulocytes (Table IX). This increase in reticulocytes was so irregular that the average curve was totally unlike that obtained with intramuscular injections, and showed a peak on the 9th day, with a percentage increase of only 73 (individual limits 8 to 256 per cent).

(c) Anacidity gastric juice. Gastric juice from patients having a constant gastric anacidity, i.e. secreting gastric juice following histamine stimulation with a $\mathrm{pH}$ less than 3 as shown by Töpfer's reagent (dimethyl-amino-azo-benzene) and no demonstrable blood or gastro-intestinal disease, was tested similarly on guinea pigs. The gastric juice was all injected intramuscularly, and the rise in reticulocytes resembled closely that obtained following the injection of normal gastric juice (Figure 2 and Table VI, average increase 240 per cent, peak on the 7 th day, individual in- creases 125 per cent to 4,600 per cent). The amounts injected ranged from $1 \mathrm{cc}$. to $0.02 \mathrm{cc}$. filtered gastric juice. Not all the animals reacted when $0.01 \mathrm{cc}$. of gastric juice was injected.

(d) The intramuscular injection of 0.5 to 0.2 cc. $\mathrm{N} / 10 \mathrm{HCl}$ caused no reticulocytosis whatsoever (Figure 3, Table VIII).

(e) Pernicious anemia gastric juice. Gastric juice was obtained from patients who had typical Addisonian anemia. They had been followed for varying lengths of time in the out-patient clinic, and were found to respond typically to the administration of liver extract. These patients were all at the beginning of a relapse and, with the exception of one, had had no treatment during the preceding three months. This one had received $3 \mathrm{cc}$. of liver extract (equivalent to 300 grams whole liver) intramuscularly the day before the juice was obtained.

Amounts varying from $1 \mathrm{cc}$. to $0.2 \mathrm{cc}$. of filtered gastric juice were injected intramuscularly into reacting guinea pigs. Except in one case, there was no reticulocytosis in any case for the first 7 days following the injection, but in 4 out of 12 cases there was a slight rise in reticulocytes on the 11th and 12th days, to drop again at or below the previous level on the 14th day (Figure $2)$. In this one exception there was a slight rise on the 5th day, to be followed by a drop, then a greater rise on the 12 th day (Table VII). The 
TABLE IX

Reticulocyte response in guinea pigs following intramuscular injection of $\mathrm{N} / 10$ hydrochloric acid

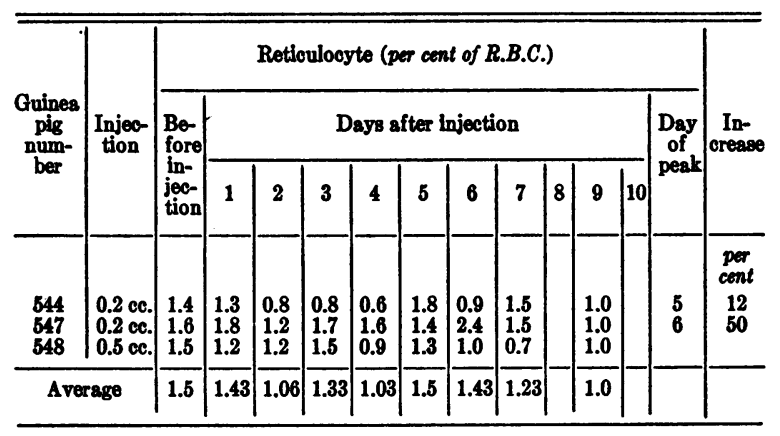

two other guinea pigs, injected with the same material, gave a response no greater than would be found in an uninjected animal. All the guinea pigs, however, responded promptly to intramuscular injections of 0.1 to $0.25 \mathrm{cc}$. of normal gastric juice administered on the 11 th to 14 th day with a reticulocyte peak on the 6 th to 7 th following this second treatment.

TABLE $X$

Summary of experimental data

\begin{tabular}{|c|c|c|c|c|c|c|c|c|}
\hline $\begin{array}{c}\text { Num- } \\
\text { ber } \\
\text { of } \\
\text { ant- } \\
\text { mals }\end{array}$ & $\begin{array}{c}\text { Material } \\
\text { used }\end{array}$ & Amount & Route* & $\begin{array}{l}\text { Aver- } \\
\text { age } \\
\text { reticu- } \\
\text { locytes } \\
\text { at be } \\
\text { ginning }\end{array}$ & $\begin{array}{c}\text { Aver- } \\
\text { sge } \\
\text { reticu- } \\
\text { locytes } \\
\text { at } \\
\text { peak }\end{array}$ & $\begin{array}{c}\text { Average } \\
\text { per- } \\
\text { centage } \\
\text { increase }\end{array}$ & $\begin{array}{c}\text { Aver- } \\
\text { age } \\
\text { day } \\
\text { of } \\
\text { peak }\end{array}$ & $\begin{array}{l}\text { Num- } \\
\text { ber } \\
\text { of } \\
\text { pa- } \\
\text { tients }\end{array}$ \\
\hline 5 & & & & 0.85 & 1.36 & 90 & & \\
\hline 4 & $\begin{array}{l}\text { Liebig's } \\
\text { beef } \\
\text { extract }\end{array}$ & $0.5 \mathrm{grams}$ & I.m. & 0.65 & 1.2 & 90 & & \\
\hline 28 & $\begin{array}{l}\text { Anti- } \\
\text { pernicious } \\
\text { anemia } \\
\text { liver } \\
\text { extract }\end{array}$ & $\begin{array}{l}0.1 \mathrm{cc} . \\
\text { (3.3 grams } \\
\text { liver) }\end{array}$ & I.m. & 0.94 & 5.06 & 440 & 6 & \\
\hline 6 & $\begin{array}{l}\text { Congo } \\
\text { red }\end{array}$ & $\begin{array}{l}225 \text { mgm. } \\
\text { in } \\
6 \text { doses }\end{array}$ & I.p. & 1.15 & 3.58 & 211 & 7 & \\
\hline 20 & $\begin{array}{l}\text { Normal } \\
\text { gastric } \\
\text { juice }\end{array}$ & $\begin{array}{l}4 \text { cc. to } \\
0.01 \mathrm{oc} .\end{array}$ & I.m. & 1.65 & 4.54 & 175 & 6 & 8 \\
\hline 8 & $\begin{array}{l}\text { Normal } \\
\text { gastric } \\
\text { juice }\end{array}$ & $\begin{array}{l}0.5 \text { ce. to } \\
0.1 \mathrm{cc} \text {. }\end{array}$ & I.p. & 1.2 & 2.02 & 73 & 11 & 4 \\
\hline 3 & $\mathrm{~N} / 10 \mathrm{HCl}$ & $\begin{array}{l}0.5 \mathrm{co} \text {. to } \\
0.2 \mathrm{ce} .\end{array}$ & I.m. & 1.3 & 1.4 & 7.5 & & \\
\hline 13 & $\begin{array}{l}\text { Anacidity } \\
\text { gastric } \\
\text { juice }\end{array}$ & $\begin{array}{l}1.0 \mathrm{cc} \text {. to } \\
0.01 \mathrm{cc} .\end{array}$ & I.m. & 1.21 & 4.13 & 240 & 7 & 6 \\
\hline 12 & $\begin{array}{l}\text { Pernicious } \\
\text { anemia } \\
\text { gastric } \\
\text { juice }\end{array}$ & $\begin{array}{l}1.0 \mathrm{cc} \text {. to } \\
0.25 \mathrm{cc} .\end{array}$ & I.m. & 1.07 & 2.8 & 170 & 9 & 6 \\
\hline
\end{tabular}

* I.m. = Intramuscular.

I.p. = Intraperitoneal.
Guinea pigs which had received injections of normal gastric juice and anacidity gastric juice did not respond further to an injection of liver extract or normal gastric juice on the 12th to 16th day, thus differing from the animals who received gastric juice from patients with pernicious anemia. The slight rise on the 12th day occurring in some of the latter animals is evidently due to a different mechanism, and is not a reaction in the sense given here.

\section{DISCUSSION AND CONCLUSIONS}

Several investigators have observed that guinea pigs respond by a reticulocytosis to injection (intramuscular, intraperitoneal or subcutaneous) of liver extract potent in the treatment of Addisonian anemia. We have obtained similar findings and have used this means of separating guinea pigs into two groups-reactors and non-reactors, the latter being discarded.

The same animals (reactors) were found to give a reticulocyte response following intraperitoneal injections of 1.5 per cent Congo red in 6 per cent glucose. The reaction was not quite so marked as that obtained with intramuscular liver extract, but it was similar in type and the animals had no further reticulocytosis following an injection of liver extract administered on the 16th day after the beginning of the Congo red treatment. Massa and Zolezzi (5) have recently confirmed this reaction in guinea pigs. Congo red further resembles liver extract in that it can be used to produce a remission in cases of pernicious anemia (6) (7). Others have failed to confirm this (23).

Castle and his coworkers (8) (9) showed that normal human gastric juice, incubated with beef muscle or with alcoholic extract of yeast, was able to produce a remission in patients suffering from pernicious anemia. The same substances have recently been shown to be reticulocytogenic for guinea pigs following oral administration (2) (4). Neither group of workers (4) (8) was able to obtain results with gastric juice alone. However, very recently Greenspon (10) produced a reticulocytosis in a pernicious anemia patient by feeding him neutralized and chilled normal gastric juice. The author felt that the neutralization and chilling inactivated the pepsin, which inactivated the antianemic factor present in gastric juice. 
Furthermore, Morris and coworkers (11) and Fouts, Helmer and Zerfas (12) have shown that intramuscular injections of large amounts of normal gastric juice, concentrated, caused a remission in pernicious anemia patients.

Singer (13) reported that rats respond to a single intramuscular injection of 1 to $20 \mathrm{cc}$. of normal, unheated gastric juice with an increase in reticulocytes, but that no such reticulocytosis was obtained with gastric juice from pernicious anemia patients. Fleischhacker and Schlesinger. (14) also working with rats obtained a similar increase in reticulocytes following the injection of hydrochloric acid and pepsin, normal gastric juice or gastric juice from 50 per cent of the cases of pernicious anemia.

Salah (15), using Singer's method, induced a reticulocytosis in rats by giving injections of gastric juice from pellagrous patients. The advisability of using rats as test animals, however, has been questioned by Engberding (16) who showed that rats have a reticulocytosis following the mere taking of blood necessary for the counts.

The intramuscular injection of gastric juice in guinea pigs gave results similar to those reported by Singer with rats (13), but there was no response to tenth normal hydrochloric acid. The normal gastric juice and that from patients having an anacidity caused a reticulocytosis, while that from pernicious anemia patients gave no immediate response. The response from the gastric juice from pernicious anemia patients occurred only in a fourth of the cases, and at a later time, 11 th to 12th day, than that found with normal gastric juice, and was not classed, therefore, as a true response. The minimal amounts necessary for evoking a response were practically the same with anacidity as with normal gastric juice, but the height of the reticulocytosis was slightly lower, indicating that the concentration of the reticulocytogenic agent was slightly less in anacidity than in normal gastric juice. None of the patients with gastric anacidity showed any anemia. Hartfall and Witts (17) testing the content of Castle's ferment in patients with simple achlorhydric anemia found that the amount present was less than in normal gastric juice, and quite variable in different patients.

The greater response with normal gastric juice cannot be attributed solely to the presence of the acid, since the intramuscular. injections of fairly large doses of tenth normal hydrochloric acid caused no reticulocytosis whatsoever.

There is no adequate explanation for the difference in action following intraperitoneal and intramuscular injection of gastric juice. The inability of normal gastric juice to be hematopoietically active when administered orally was attributed by Castle and his coworkers to the absence of an extrinsic factor to react with the intrinsic factor present in the gastric juice. The extrinsic factor was thought to be present in muscle and alcoholic extract of yeast, used in the incubation. Greenspon (10) feels that this lack of activity is due to antagonistic action of the pepsin towards the antianemic principle in the gastric juice. The pepsin is removed by absorption on muscle, or rendered inactive by chilling and neutralizing the gastric juice. Following this treatment, normal gastric juice has been stated to be hematopoietically active when administered orally, though it may have reacted with food eaten four hours later.

The results of Fouts et al. (12), on the other hand, fail to show any injury to the antianemic principle due to concentrating whole gastric juice in vacuo at $40^{\circ} \mathrm{C}$., the temperature at which pep$\sin$ is most active.

The gastric juice was passed through an ultrafilter which held back both the pepsin and rennin. When the gastric juice was merely preserved in the ice box for two months before filtering, the concentrate alone had antianemic properties, while the filtrate was totally inactive. This showed that untreated active principle was not able to pass through the filter.

When, however, the material had been concentrated in vacuo at $40^{\circ} \mathrm{C}$. both the filtrate and concentrate had antianemic properties, indicating either a physical or chemical change in the antianemic substance. That such a change must have taken place is further demonstrated by the fact that with fresh gastric juice neither the filtrate nor the concentrate had any hematopoietic activity.

Braun (18) suggested that gastric juice is not effective by mouth because the active principle does not pass ultrafilters and therefore cannot be absorbed from the gastro-intestinal mucosa. The 
digestion with beef muscle, yeast extract or concentration in vacuo at $40^{\circ} \mathrm{C}$. presumably causes a reaction between this active principle and a protein molecule, which then acts as a conveyor of the active principle through the mucous membranes. Braun (18) cites as corroborative evidence the fact that Congo red does not pass ultrafilters until attached to a protein molecule (19). Since we found that Congo red was hematopoietic when injected intraperitoneally, it is probable that the irregular reticulocytosis following intraperitoneal injections of active juice was not due solely to lack of absorption. One-fiftieth the amount of gastric juice injected intraperitoneally causes a reticulocytosis following intramuscular injection.

Since the same response was obtained following intramuscular injections of normal and anacidity gastric juice and with Congo red intraperitoneally, it is not possible to prove with certainty that the reticulocytogenic substance of gastric juice is identical with Castle's ferment.

Gastric juice from patients with Addisonian anemia contains less than one-fiftieth the amount of reticulocytogenic substance present in normal gastric juice.

Barnett (20), Castle et al. (21) and Beebe and Wintrobe (22) have used the demonstration of the presence or lack of Castle's "intrinsic" factor as a diagnostic test in cases of obscure anemia by incubating with beef muscle the gastric juice from the patient to be investigated and administering the mixture to a case of pernicious anemia. This test, however, took large amounts of gastric juice, and the presence of a known case of pernicious anemia in relapse. The results of injecting gastric juice intramuscularly into guinea pigs are just as striking as the administering of this gastric juice-beef muscle preparation to the patient, and more easily obtainable. We suggest that this reaction of the guinea pig be used as a diagnostic procedure in cases of obscure anemia. The amount of gastric juice necessary is very small, 1 to $2 \mathrm{cc}$. being ample. It is essential, however, to have the gastric juice free from bile; as otherwise large areas of necrosis are caused with amounts as small as $0.2 \mathrm{cc}$., and frequently death on the third or fourth day with larger amounts.

\section{SUM MARY}

The presence of a substance in antipernicious anemia liver extract, reticulocytogenic for the majority of guinea pigs, was confirmed. A similar substance was demonstrated following intraperitoneal injection of Congo red and intramuscular injection of gastric juice from normal individuals, and also from patients having gastric anacidity but no anemia.

Irregular and late reticulocytosis was obtained following intraperitoneal injection of normal gastric juice. The mechanism of this altered activity is not clear.

The intramuscular injection of gastric juice from patients suffering from typical Addisonian anemia caused no reticulocytosis within the first 8 days. This reaction of the guinea pig to the intramuscular injection of gastric juice is proposed as a test in the diagnosis of anemia of obscure origin.

\section{BIBLIOGRAPHY}

1. Jacobson, B. M., On a pernicious anemia-like state in the guinea pig. J. Clin. Invest. (Proc.), 1934, 13, 714.

2. Jacobson, B. M., The response of the guinea pig's reticulocytes to substances effective in pernicious anemia. J. Clin. Invest., 1935, 14, 665.

3. Landsberg, J. W., and Thompson, M. R., The guinea pig as a hematopoietic test animal. J. Am. Pharm. A., 1934, 23, 964.

4. Miller, D. K., and Rhoads, C. P., The reticulocyte response in guinea pigs following the oral administration of certain anti-anemic substances. New England J. Med., 1935, 213, 99.

5. Massa, M., and Zolezzi, G., Personal communication to the author.

6. Massa, M., and Zolezzi, G., Die Wirkung des Kongorotes bei perniziöser Anämie. Klin. Wchnschr., 1935, 14, 235.

7. Mermod, C., and Dock, W., A colloidal dye effective in treating pernicious anemia and evoking reticulocytosis in guinea pigs. Science, 1935, 82, 155.

8. Castle, W. B., and Townsend, W. C., Observations on the etiologic relationship of achylia gastrica to pernicious anemia. II. The effect of the administration to patients with pernicious anemia of beef muscle after incubation with normal human gastric juice. Am. J. M. Sc., 1929, 178, 764.

9. Strauss, M. B., and Castle, W. B., The nature of the extrinsic factor of the deficiency state in pernicious anemia and in related macrocytic anemias. New England J. Med., 1932, 207, 55. 
10. Greenspon, E. A., The nature of the antipernicious anemia principle in stomach. I. Method to improve stomach preparations. J. A. M. A., 1936, 106, 266.

11. Morris, R. S., Schiff, L., Burger, G., and Sherman, J. E., A specific hematopoietic hormone in normal gastric juice. J. A. M. A., 1932, 98, 1080.

12. Fouts, P. J., Helmer, O. M., and Zerfas, L. G., The formation of a hematopoietic substance in concentrated human gastric juice. Am. J. M. Sc., 1934, 187, 36.

13. Singer, $K$., Uber eine tierexperimentelle Methode zum Nachweis des Castle-Prinzips des Magensaftes und deren klinische Bedeutung. Klin. Wchnschr., 1935, 14, 200.

14. Fleischhacker, H., and Schlesinger, A., Retikulozytenkrisen bei Ratten nach Injektion von Magensaft. Med. Klin., 1935, 31, 182.

15. Salah, M., The demonstration of the haematopoietic principle in chronic pellagric achylia. Tr. Roy. Soc. Trop. Med. and Hyg., 1935, 29, 299.

16. Engberding, J., Der Einfluss von Magensaft auf die Blutbildung bei Ratten. Monatschr. f. Kinderh., 1934, 59, 332.
17. Hartfall, S. J., and Witts, L. J., The "intrinsic factor" of Castle in simple achlorhydric anemia. Guy's Hosp. Rep., 1933, 83, 24.

18. Braun, B., Der Mechanismus der therapeutischen Einwirkung des Castleschen Prinzips bei der Perniziosen Anämie. Fol. haemat., 1934, 53, 27.

19. Bennhold, H. (Quoted by Braun), Med. Kolloidlehre, Lichwitz-Liesegang-Spiro, Dresden-Leipzig. 1933, p. 474.

20. Barnett, C. W., The significance of the gastric secretions in pernicious anemia. Am. J. M. Sc., 1931, $182,170$.

21. Castle, W. B., Heath, C. W., and Strauss, M. B., A biologic assay of the gastric secretion of patients with pernicious anemia having free hydrochloric acid and that of patients without anemia or with hypochromic anemia having no free hydrochloric acid, and of the rôle of intestinal impermeability to hematopoietic substances in pernicious anemia.

22. Beebe, R. T., and Wintrobe, M. M., Diagnosis of obscure cases of pernicious anemia. Arch. Int. Med., 1933, 51, 630.

23. Barker, H., Treatment of pernicious anemia with Congo red. J. Clin. Invest. (Proc.), 1936, 15, 456. 